\title{
Data Acquisition Enhancement in Shape and Multispectral Color Measurements of 3D Objects
}

\author{
Grzegorz Mączkowski, Robert Sitnik, and Jakub Krzesłowski \\ Institute of Micromechanics and Photonics, \\ Warsaw University of Technology \\ 8 Boboli, Warsaw, PL 02-525 \\ $\mathrm{g}$. maczkowski@mchtr.pw.edu.pl \\ http://ogx.mchtr.pw.edu.pl/
}

\begin{abstract}
The paper presents application of a correction technique proposed for registering images by a CCD (Charged Coupled Device) camera. The device is installed in a $3 \mathrm{D}$ shape, angular reflectance distribution and multispectral color measurement system set up for digitization of cultural heritage objects. The procedure compensates for the camera noise and scene illumination non-uniformity according to previously published model. The paper describes the measurement system and provides analysis of data collected from measurements of the Color Checker reference target and uniform reference plane to evaluate enhancement of reflectance and shape accuracy after correction. Additionally a few examples of digitized objects are shown.
\end{abstract}

\section{Introduction}

Digitization of cultural heritage objects is recently becoming more available and well known in artifacts conservation. However, to better use its potential possibilities and to achieve truthful results, it is necessary to understand principles of image acquisition techniques and tune them, so that measurement uncertainty can be reduced.

The measurement process usually assumes determination of shape of the surface by the means of a 3D scanner and additionally its color using RGB or multispectral camera. Known solutions are based on laser scanning devices 1] as well as the ones using structured light projection technique[2 3] and usually a multi-band, self made camera with interference filters to separate spectral channels 14. Some systems use a single detector for data acquisition, so that there is no need to manually align color texture to cloud of points which represents shape 3], but there are also solutions which require some manual adjustment 4. Despite construction differences all devices known by the authors are based on non-contact data acquisition by capturing images with a digital camera. Therefore it is important to consider the capturing process and eliminate errors characteristic for digital image registration, such as noise and non-uniform illumination. Very thorough examination of this problems can be found in work 5 . 
This paper describes the application of method presented in work [5] to a developed integrated measurement system which includes $3 \mathrm{D}$ shape measurement system using structured light projection method, multispectral 10 band camera for color measurement and additional directional illumination setup for establishing surface reflectivity in a sense of BRDF (Bidirectional Reflectance Distribution Function) model. First the measurement setup is described, following brief outline of image acquisition method with some implementation details. After that evaluation results of shape and color measurement are described and commented.

\section{Measurement Setup}

The 3D shape measurement method uses the 3D Measurement with Algorithms of Directional Merging And Conversion (3DMADMAC) system [6]. This method of measurement is based on a structured light technique with digital sine patterns and Gray codes projection (Fig. 2 a). The system consists of a Digital Light Projector (DLP) and an industrial CCD camera (Figure 2a). The 3DMADMAC system can be customized depending on end user requirements regarding size of measurement volume, amount of measurement points and duration of a single measurement. It also consists of a set of Software Development Kit tools which extend its functionality and automate all required measurement and data analysis algorithms.

Color of a measured surface is captured using a multi-spectral approach. The custom built camera was constructed to register images in 10 spectral bands with the aid of interference filters (Fig. 2b). The filter wheel is placed between the camera matrix and the lens. It has 11 slots, because additional empty window without a filter is necessary for performing shape and BRDF measurement which uses the same detector. The lens mount is located outside the case which allows for simple lens replacement according to required measurement conditions.

Normally the multispectral capture system uses analytic calibration procedure described in previous work 7 . It is based on capturing images of white reference plate for light source spectrum compensation and images of uniform background to compensate for illumination distribution and spectral filters' angular characteristic. Additionally transverse shifts of images due to positioning errors can be eliminated. For the purpose of the described analysis the spectral images calculation procedure was modified in order to properly perform new image acquisition process.

Another device is employed to measure angular reflectance distribution of surface. It comprises of a set of light sources distributed on a grid pattern and illuminating measurement volume. Each illuminator resembles a lambertian source and allows directional illumination of the investigated surface (Fig. 2b). Pictures are captured with all illuminators turned on sequentially which gives a collection of reflectance values in the function of illumination angle and serves as a BRDF estimation which leads to Phong parameters calculation [8]. The result is a BRDF function modeled with Phong parameters[9]. This paper however, will not further discuss analysis of angular reflectance data. 

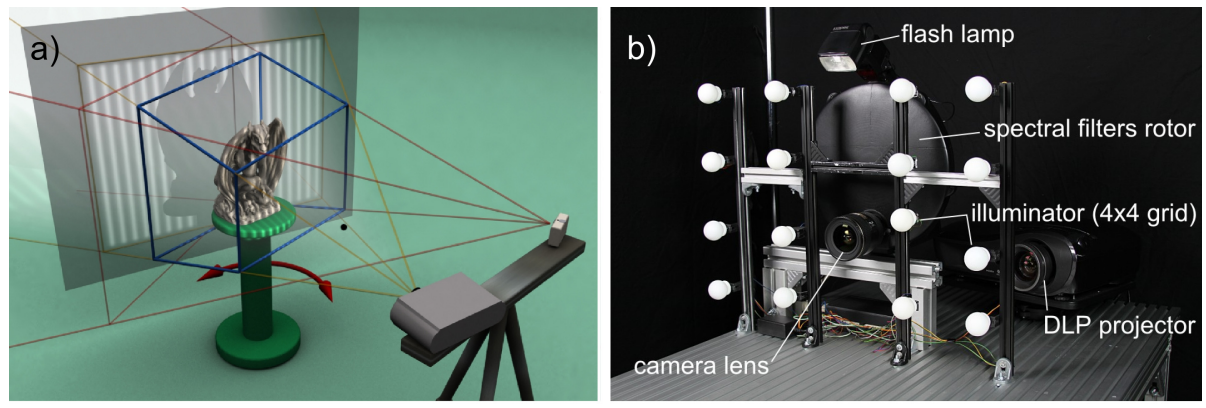

c)



d)

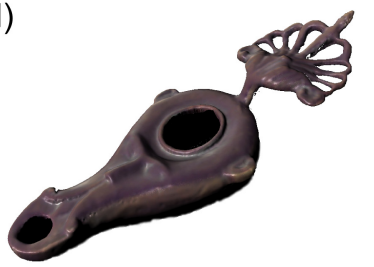

e)

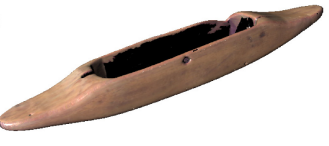

f)

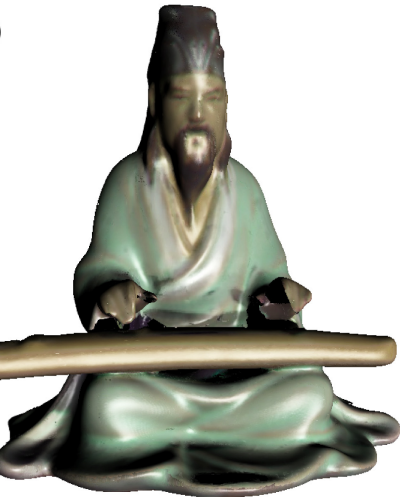

Fig. 1. Measurement system and virtual models of previously digitized objects: a) concept of the shape measurement device; b) constructed measurement setup; c) painted, wood-carved Nativity Scene figure; d) brass olive lamp; e) wooden shuttle on a loom; f) china figure

\section{Data Acquisition Procedure}

The goal of presented research was to implement and adopt a concept proposed by Mansouri et. al. in [5] of a digital camera calibration to reduce noise and nonuniformity of illumination. The purpose was to enhance data acquisition process in the case of the multispectral camera and 3D scanner. The proposed solution is based on the camera response in each pixel according to the following model described by equation (11).

$$
[R]=[O]+[T]+[U \times S]
$$

The camera response $[R]$ is a sum of a zero level (offset) image $[O]$, thermal signal proportional to acquisition time $[T]$ and a product of useful signal $[U]$ and sensor response $[S]$. Further reasoning leads to derivation of a formula for the useful 
signal, knowing the offset, the thermal and the flat field $[F]$ characteristics, as in formula (2). For details see[5].

$$
[U]=\frac{[R]-[O]-[T]}{[F]-[O]-\left[T_{F}\right]} \times F_{\text {mean }}
$$

Implementation of the proposed model required acquisition of additional calibration data (offset, thermal and flat field images) and including them in data processing path. They are registered for every shutter value used in the measurement. Additionally the radiometric calibration procedure $(R C)$ calculates mean values for flat field images which are afterwards used to rescale the useful images into proper range.

Because the software controlling the measurement system has a modular structure it was possible to implement an adapter for a detector module which is responsible for preparing raw images for further processing, without modification of the measurement head module. The only modification was made to the module, which calculates spectral reflectance, because it already used flat field image and it was not desirable to take it into account twice. Therefore the color measurement procedure benefits mainly due to camera noise reduction. The detector adapter module averages several frames to reduce temporal noise and applies compensation procedure mentioned above before sending data further along the processing path.

The camera installed in the measurement system is a Prosilica GE4900 device with 16Mpix sensor equipped with 12bit Analog-to-Digital Converter (ADC), which allows for increased signal quantization resolution, especially important for capturing spectral images. The camera was additionally tested for its linearity by the comparison of its response with a light meter. Results show a 0.99992 coefficient of cross correlation with the best fitted plane and root mean square error of $2.12 \%$ in relation to measured intensity range. Based on these results the decision was made to do not perform additional non-linearity correction.

Illumination for color measurement was provided by a digital light projector with a halogen lamp, which was also used for structured light projection for shape measurement. This allowed for a single procedure for flat field image acquisition for both measurement modules. Moreover the DLP projector's light flux is quite stable after sufficient warm up time, so there is no meaningful drift of spectral images lightness. Measurements were conducted in a controlled laboratory environment, after stabilization of thermal conditions for electronic devices and measurement volume.

\section{Evaluation of Color Measurement Results}

Evaluation of color measurement system accuracy was based on measurement of Color Checker target which was scanned with $(R C+)$ and without radiometric calibration $(R C$-). Spectral reflectance data registered from both approaches was averaged for every color patch and compared with each other and with reference 
measurement of the color target made with a Minolta CM-2600D spectrophotometer. Root mean square deviations from reference measurement for $R C_{+}$ and $R C$ - were calculated for each color patch and are presented in Fig. 2, Measurement of two patches was not possible because of too low contrast of fringes projected during shape measurement. Consequently there was no sufficient phase information to calculate cloud of points for these patches. A few patches, indicated with a black border came out worse with the $R C+$ procedure, which is especially visible for the neutral ones. Our initial assumption was that it is the result of not implemented correction for camera non-linearity, but most likely this outcome is due to quantization noise which varies between spectral channels. It is the effect of a fixed shutter value for all channels and different transmission of spectral filters. Consequently the exposure of spectral images varies and so does the quantization noise. It is planned in the next step of research to implement constant exposure conditions by adjusting of shutter value for all spectral channels independently.

Fig. 3] shows spectral reflectance measurement results of sample color patches. Analysis of the reflectance plots leads to the conclusion that measurement errors are distributed along the whole registered spectrum and do not occur in any specific wavelength. However there is a trend that the difference between reference and estimated reflectance is bigger for small reflectance values, which agrees with the mentioned assumption that the quantization noise affects the camera response.



Fig. 2. RMS values for Color Checker patches measured with $R C+$ (bold font) and $R C$ (normal font) procedure compared with the reference spectrophotometer measurement. Patches with black border are worse with $R C+$ comparing with $R C$-.

Comparison of color obtained in sRGB[10] color space shows that $R C+$ measurement gives more uniform output, with less chromatic noise and overall better hue than $R C$ - (Fig. 4). 




Fig. 3. Spectral reflectance plots for chosen color patches

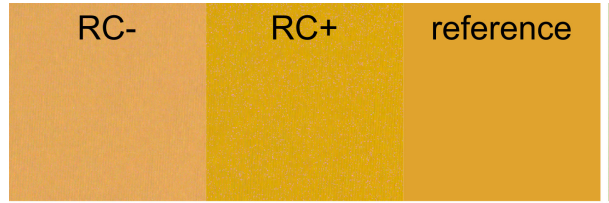

orange yellow patch

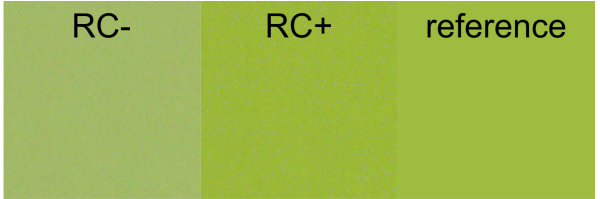

yellow green patch

Fig. 4. Comparison of sRGB color values for exemplary target patches

\section{Evaluation of Shape Measurement Results}

The shape measurement uses sinusoidal fringe projection by the means of a DLP projector. Phase of fringes is established by the temporal phase shifting algorithm 11 in several reference planes within a measurement volume. Afterwards it is interpolated over the whole calibrated space. The algorithm gives very accurate results, but is sensitive to differences of lightness between consecutive fringe images and variations in phase shifts. In this case the latter are negligible, because the DLP projector has very high repeatability when the period of fringes is an integer multiple of number of projector pixels. Nevertheless image noise influences phase calculation accuracy and non-uniform illumination causes varying contrast of fringes within a single image. The same radiometric 
calibration procedure was therefore applied to correct the fringe images to improve phase quality.

To evaluate results of this improvement two measurements of a uniform plane reference target of size $300 \times 220 \mathrm{~mm}$ were conducted with and without radiometric calibration, so that deviations from an ideal fitted plane could be compared in both situations. The obtained clouds of points have on average 100 points per square millimeter. Fig. 5 visualizes error distribution after plane fitting to measurement data. It shows that in each interval of one, two and three standard deviations the plane fit error is 1.5 to 2 times smaller after the application of radiometric calibration procedure.

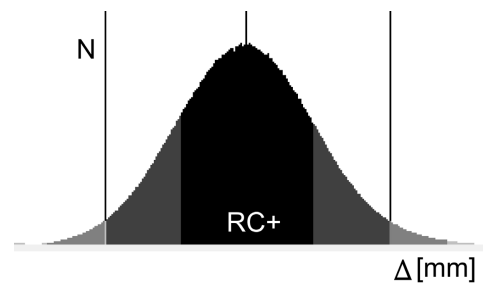

error for $66 \%$ of points: $0.01605 \mathrm{~mm}$ error for $95 \%$ of points: $0.03456 \mathrm{~mm}$ error for $99 \%$ of points: $0.04855 \mathrm{~mm}$ maximum error:

$0.10533 \mathrm{~mm}$

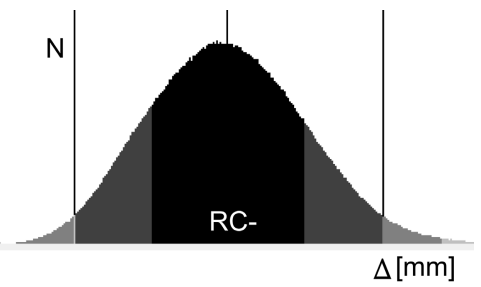

error for $66 \%$ of points: $0.02774 \mathrm{~mm}$ error for $95 \%$ of points: $0.05609 \mathrm{~mm}$ error for $99 \%$ of points: $0.07766 \mathrm{~mm}$ maximum error: $\quad 0.15779 \mathrm{~mm}$

Fig. 5. Comparison of plane fit error distribution. The plot shows error value $\Delta$ around fitted plane versus number of points $(\mathrm{N})$.

Fig. 6] shows comparison between registered corresponding fringe images and local fringes contrast values. It is visible that after radiometric correction contrast variation is less pronounced compared to the case without enhancement.


Fig. 6. Comparison of fringe images and their local contrast 


\section{Conclusions}

Presented work shows application of a digital camera response model to image acquisition procedure in an integrated shape and reflectance measurement system. It shows that such enhancement leads to more accurate measurement results, especially for spectral and fringe images. It gives foundation for future research which may include application of this method for capturing images for BRDF measurement and implementation of constant exposure in different spectral channels which will further improve registered data.

It is necessary to point out that in case of $3 \mathrm{D}$ measurement the flat field image compensation is generally not sufficient to eliminate influence of uneven illumination, because in such case it is necessary to consider more complex illumination pattern. The measured object occupies certain volume in space, so points on its surface are placed in different distances from the detector and not on a single plane as it is assumed for flat field image compensation. Additionally different points on the measured surface may have different reflection properties, which will influence spectral acquisition. This introduces opportunity for future work, because solving the mentioned problems will be very important for faithful digitization of cultural heritage objects.

Acknowledgments. This work was performed under the grant No. PL0097 financed by the Norwegian Financial Mechanism and EEA Financial Mechanism (2004-2009) and partially under the statutory work of Warsaw University of Technology.

\section{References}

1. Tonsho, K., Akao, Y., Tsumura, N., Miyake, Y.: Development of gonio-photometric imaging system for recording reflectance spectra of 3 d objects. In: Proc. SPIE, vol. 4663 , pp. 370-378 (2002)

2. Sitnik, R., Mączkowski, G., Krzesłowski, J.: Calculation Methods for Digital Model Creation Based on Integrated Shape, Color and Angular Reflectivity Measurement. In: Ioannides, M., Fellner, D., Georgopoulos, A., Hadjimitsis, D.G. (eds.) EuroMed 2010. LNCS, vol. 6436, pp. 13-27. Springer, Heidelberg (2010)

3. Mansouri, A., Lathuiliere, A., Marzani, F., Voisin, Y., Gouton, P.: Toward a 3d multispectral scanner: an application to multimedia. IEEE Multimedia 14(1), 40-47 (2007)

4. Simon, C., Huxhagen, U., Mansouri, A., Heritage, A., Boochs, F., Marzani, F.: Integration of high resolution spatial and spectral data acquisition systems to provide complementary datasets for cultural heritage applications. In: Proc. SPIE, vol. 7531(1), p. 75310L (2010)

5. Mansouri, A., Marzani, F.S., Gouton, P.: Development of a protocol for ccd calibration: Application to a multispectral imaging system. International Journal of Robotics and Automation 20(2), 94-100 (2005)

6. Sitnik, R., Kujawinska, M., Woznicki, J.: Digital fringe projection system for largevolume 360-deg shape measurement. Opt. Eng. 41, 443-449 (2002) 
7. Mączkowski, G., Sitnik, R., Krzesłowski, J.: Integrated method for 3d shape and multispectral color measurement. Journal of Imaging Science and Technology 55(3), 030502-(10) (2011)

8. Mączkowski, G., Sitnik, R., Krzesłowski, J.: Integrated three-dimensional shape and reflection properties measurement system. Appl. Opt. 50, 532-541 (2011)

9. Phong, B.: Illumination for computer generated pictures. Communications of the ACM 18, 311-317 (1975)

10. IEC 61966-2-1:1999: Multimedia systems and equipment. Colour measurement and management. Colour management. Default RGB colour space. sRGB

11. Chen, F., Brown, G.M., Song, M.: Overview of three-dimensional shape measurement using optical methods. Optical Engineering 39(1), 10 (2000) 